\title{
Downstream physics of the helicon discharge
}

\section{Francis F Chen, Isaac D Sudit and Max Light}

Electrical Engineering Department, University of California, Los Angeles, California 90024-1594, USA

Received 9 October 1995, in final form 21 December 1995

\begin{abstract}
Measurements of the radial and axial profiles of both the plasma parameters and the wave properties in a long, thin helicon discharge show that most of the RF power is deposited near the antenna and that a dense, cool $\left(T_{e}<2 \mathrm{eV}\right)$ plasma can be obtained in the downstream region. The density $n$ and electron temperature $T_{e}$ profiles in that region can be explained quantitatively with classical collisional theory, and factor-of-two agreement can be obtained on total particle and energy balance. Spatial modulation of the helicon wave amplitude can be explained by the beating of two different radial modes launched simultaneously by the antenna. Though the helicon wave can be shown to be essential to the production of high densities, it plays little role in the downstream evolution of the plasma. These results indicate that helicon discharges can produce the cool plasmas normally associated with afterglows without the attendant loss of density.
\end{abstract}

\section{Introduction}

The advantages of high-density, low-pressure plasma sources, including helicon wave sources, for semiconductor processing have been enumerated by a number of authors [1-4]. Recently, two other benefits of these sources can possibly be added: the absence of particulate formation at low pressures [5] and the enhanced etch rate from negative ion formation in the afterglow when these sources are pulsed $[6,7]$.

In this paper we summarize our work on the physics of helicon discharges, especially in the downstream region far from the antenna. Helicon sources are more complex than other plasma sources because they depend on wave propagation and contain a magnetic field strong enough to constrain the electrons. For this reason, we have simplified the situation by using a uniform magnetic field and a uniform tube diameter. The experiments were done in a $5 \mathrm{~cm}$ diameter, $1.6 \mathrm{~m}$ long quartz tube filled with $15 \mathrm{mTorr}$ of argon (figure 1). The magnetic field $B_{0} \hat{z}$ of $0-1.2 \mathrm{kG}$ was set at 0 or $800 \mathrm{G}$ for most of this work, and the RF power was $2 \mathrm{~kW}$ at $27.12 \mathrm{MHz}$. Straight Nagoya Type III (N) and right-hand (R) and left-hand (L) helical antennas with $m=1$ azimuthal symmetry were used. Most of the data was taken with the R-antenna because it gave the highest density and cleanest results. Axial scans were made with different types of thin-shafted probes which could be moved inside the discharge chamber without interfering with the discharge. Details of the apparatus can be found in previous papers [8-11].

\section{Mode structure}

Identification of the helicon modes was made with radial scans of the wave magnetic field components $B_{r}, B_{\theta}$, and $B_{z}$ with a single-turn magnetic probe [9]. Figure 2(a) shows field profiles for an R-antenna compared with theoretical curves computed for the $m=+1$ and $m=-1$ circularly polarized modes taking into account the measured plasma density profile $[12,13]$. The data agree well with the $m=+1$ mode and not at all with the $m=-1$ mode. In figure 2(b), the data were taken with the magnetic field reversed. In this case, the R-antenna is expected to launch an $m=-1$ mode in the downstream direction (toward the midplane of the tube). However, it is seen that the data agree much better with the $m=+1$ mode than with the $m=-1$ mode. This is also the case with the $\mathrm{N}$-antenna. The prevalence of the $m=+1$ mode is not yet completely understood.

\section{Importance of the helicon resonance}

That propagating helicon waves are necessary for highdensity operation is demonstrated by the optical emission data shown in figure 3. A small lens connected via an optical fibre to a monochromator measures the local $488 \mathrm{~nm}$ $\mathrm{Ar}^{+}$light emitted by the plasma. In figure 3(a), the dc magnetic field $B_{0}$ is varied from 0 to $300 \mathrm{G}$. At $0 \mathrm{G}$, the discharge is a non-resonant inductively coupled plasma (ICP), and the light is confined to the antenna region. As $B_{0}$ is increased, a second peak appears downstream and grows with $B_{0}$, dominating the ICP peak at fields above $150 \mathrm{G}$. 


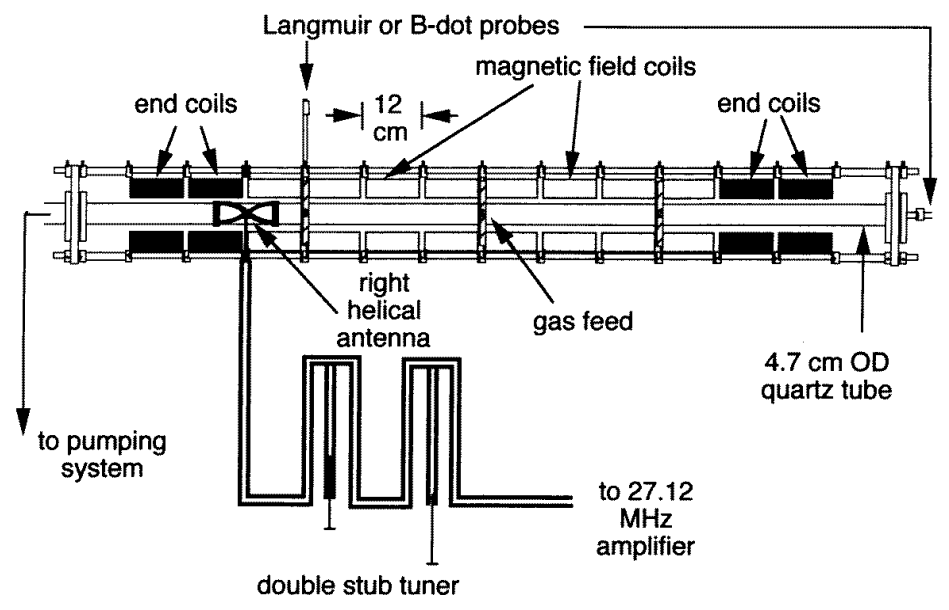

Figure 1. Schematic of the apparatus.
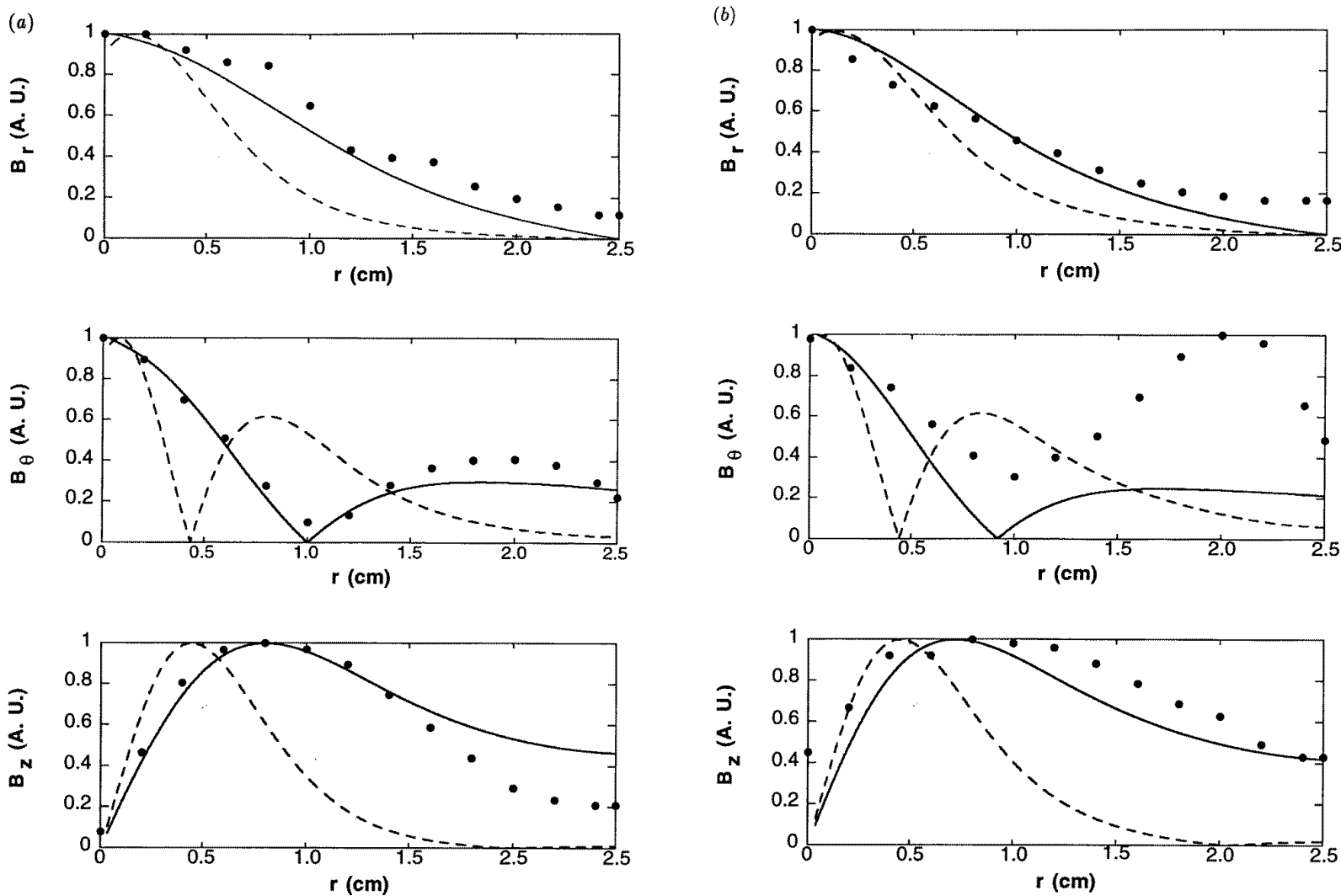

Figure 2. Radial scans of the wave magnetic field components (points), normalized to unity, in an $800 \mathrm{G}$ helicon discharge at 7.5 mTorr argon fill pressure, with excitation by an R-antenna, for (a) $\boldsymbol{B}_{0}$ parallel to $\boldsymbol{k}$ and (b) $\boldsymbol{B}_{0}$ antiparallel to $k$. The theoretical curves are for the $m=+1$ (solid line) and $m=-1$ (dashed line) modes, computed for a plasma with the measured radial density profile.

At $900 \mathrm{G}$, this peak is 30 times higher than the ICP peak. That the downstream peak is related to helicon waves is shown by figure $3(\mathrm{~b})$. The R-antenna is designed to launch an $m=+1$ helicon wave only in the direction of $\boldsymbol{B}_{0}$. In figure 3(b) it is seen that the 'downstream' direction is reversed when $\boldsymbol{B}_{0}$ is reversed, suggesting energy transport by the helicon wave. The Nagoya $\mathrm{N}$-antenna, however, is plane-polarized and therefore should excite $m=+1$ waves equally well in either direction. That this is true is seen from the symmetric peaks in figure 3(c). The asymmetry of light emission with the R-antenna also shows that the increase in density with $B_{0}$ is not merely caused by increased confinement; a directional wave is involved. Further details are given in [11]. 


\section{Axial variation of plasma parameters}

To measure the axial variation of $n, T_{e}$, and space potential $V_{s}$, a Langmuir probe was constructed with a thin glass shaft lying on the bottom of the tube and with a dogleg to bring the probe tip to the axis. The probe is compensated for RF potential fluctuations with an auxiliary floating electrode, as described in a previous paper [14]. Figure 4(a) shows the axial profiles of $n$ and $T_{e}$ at $800 \mathrm{G}$. It is seen that $T_{e}$ peaks about $10 \mathrm{~cm}$ downstream from the antenna and falls from $\sim 5 \mathrm{eV}$ to $\sim 2 \mathrm{eV}$ at $80 \mathrm{~cm}$. The density, however, rises downstream, reaching a broad peak centred at around $z=50 \mathrm{~cm}$, where $z$ is measured from the midplane of the antenna. By comparison, figure 4(b) shows the same data for a $0 \mathrm{G}$ ICP plasma. Here, $n$ is considerably lower, and both $T_{e}$ and $n$ are very small outside the antenna region. (The peak at large $z$ is spurious; we believe it is caused by the failure of RF compensation at low densities.) Figure 4(c) shows the floating $\left(V_{f}\right)$ and space $\left(V_{s}\right)$ potentials. The latter was taken as the voltage at which the derivative of the probe's I-V curve drops sharply; this value agrees with the $V_{s}$ computed from $V_{f}$ and $T_{e}$. It is clear that the parallel electric field $E_{z}$ is quite small, as expected of a highly (10\%) ionized plasma.

The surprising observation that the density peaks far from the antenna cannot be explained in terms of increased ionization or decreased loss rate there, since the $T_{e}$ behaviour would indicate the opposite. There is also no large electric field to cause ions to stream into that region, as seen from the $V_{s}$ profile. If a small population of fast electrons of, say, $150 \mathrm{eV}$ energy were created in the antenna region, these could distribute their ionizing collisions over lengths of order $50 \mathrm{~cm}$, but the density would not expected to rise with $z$ when the light emission does not. Furthermore, as we shall see later, ionization by the thermal distribution of electrons is more than sufficient to account for all the ions which are lost. Fortunately, the downstream density rise can be explained by pressure balance, as explained below.

\section{Explanation of axial profiles}

\subsection{Temperature profile}

Since $T_{e}$ peaks within one helicon wavelength of the end of the antenna, the electrons are apparently heated in the near-field of the antenna. The processes occurring there can include electron acceleration by capacitively or inductively coupled electric fields as well as by helicon wave generation and damping, including electron trapping by the wave. In this paper we do not discuss these nearfield phenomena, and the reader is referred to the work by Ellingboe et al [15]. The behaviour of $T_{e}$ downstream from the peak, however, can be predicted quantitatively by heat conduction.

In the steady state, the equation for electron heat flow is

$$
\partial q / \partial z=Q=Q_{\text {wave }}-Q_{\text {inel }}-Q_{\text {elas }}-Q_{\perp}
$$

where

$q=-\kappa_{\|} \frac{\partial}{\partial z}\left(K T_{e}\right) \quad \kappa_{\|}=3.2 \frac{n K T_{e}}{m} \tau_{e}$
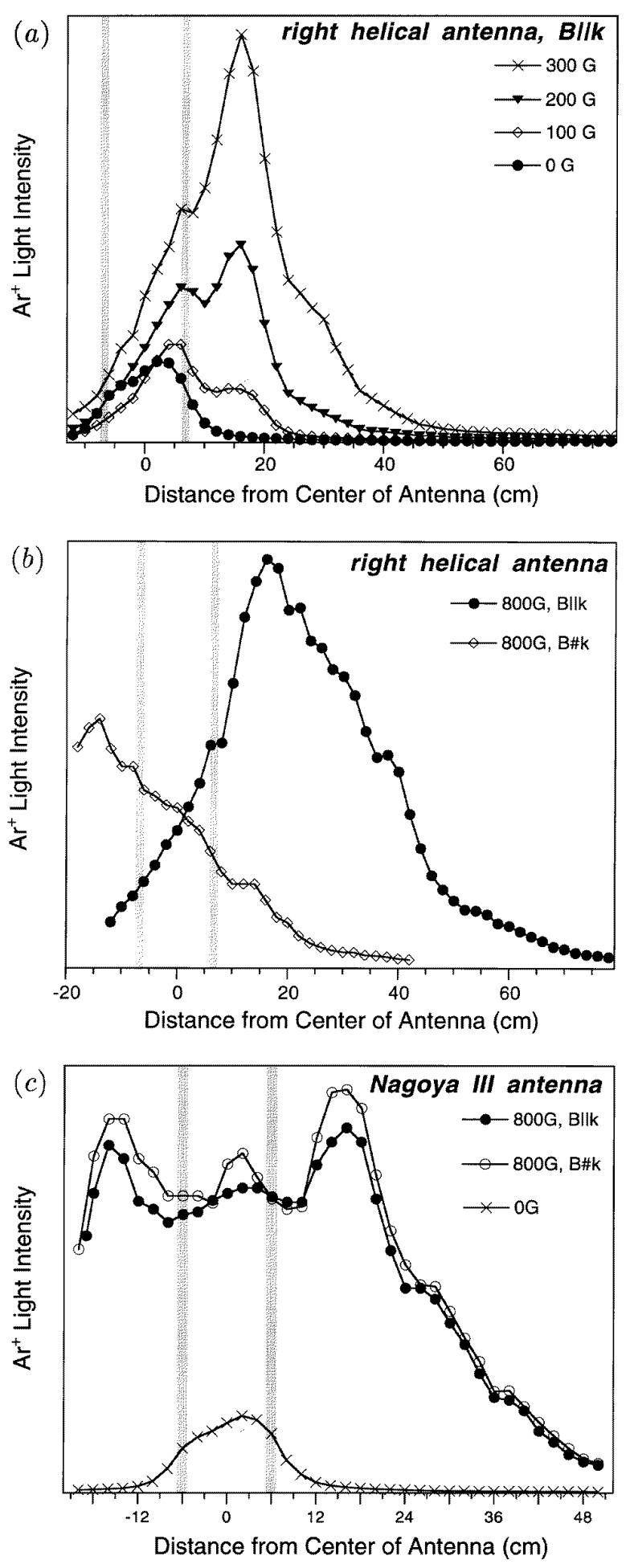

Figure 3. Optical emission measurements of the $488 \mathrm{~nm}$ $\mathrm{Ar}^{+}$line as a function distance from the antenna (shaded lines). In (a) the R-antenna is used and the magnetic field is varied from 0 to $300 \mathrm{G}$. In (b), with the $\mathrm{R}$-antenna, the $800 \mathrm{G}$ magnetic field is has the normal and reversed directions. In (c) the $\mathrm{N}$-antenna is used and the magnetic field is zero or $800 \mathrm{G}$ in either direction.

$$
\tau_{e}=3.44 \times 10^{5} \frac{T_{e V}^{3 / 2}}{n \ln \Lambda} .
$$



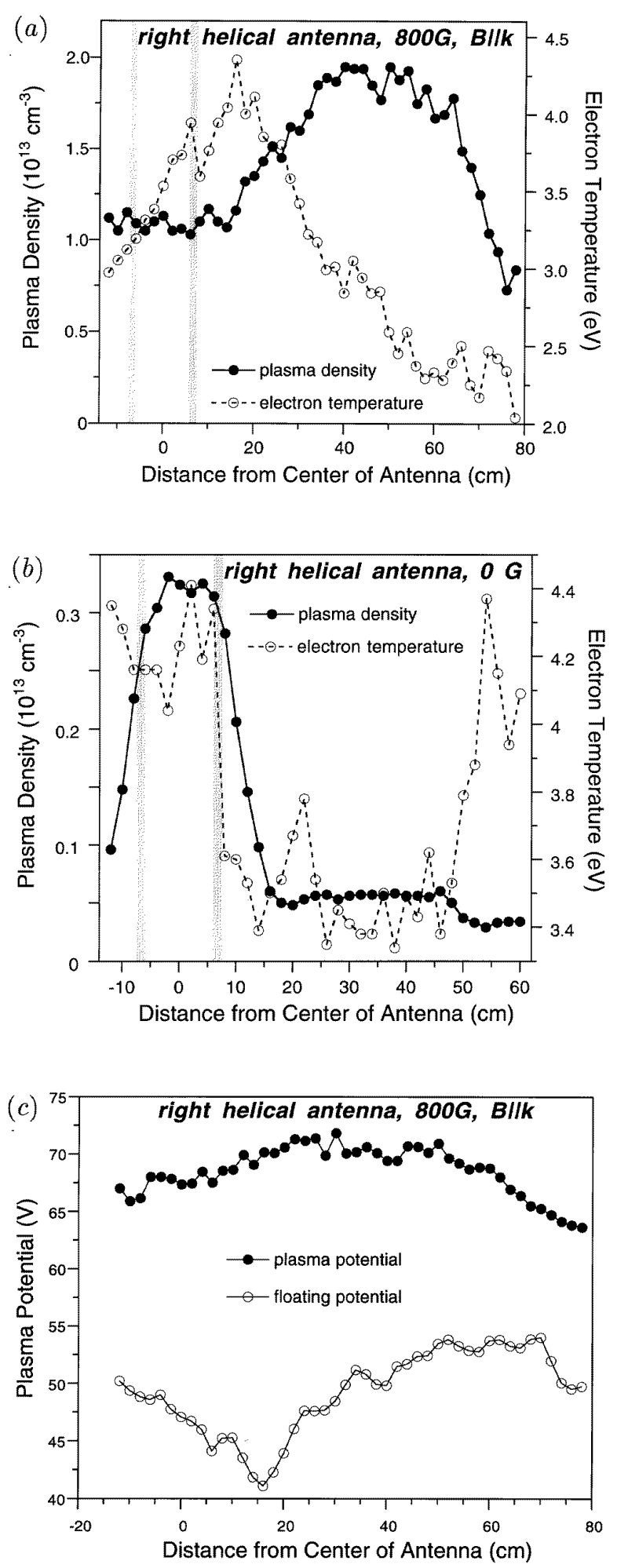

Figure 4. Measurements electron temperature and plasma density as a function of distance from the antenna (shaded lines), for (a) an $800 \mathrm{G}$ helicon discharge, and (b) a 0 G ICP discharge. In (c), the axial variation of floating potential $V_{f}$ and space potential $V_{s}$ are shown.

The heat flow $q$ is independent of $n$. The source term $Q_{\text {wave }}=\left\langle j_{z} E_{z}\right\rangle$ owing to the damping of the helicon

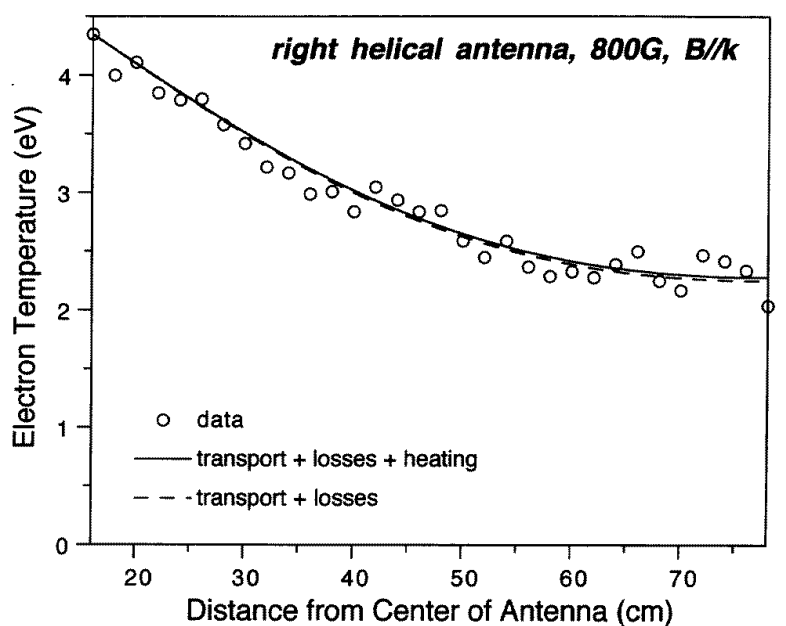

Figure 5. Computed decay of electron temperature downstream, in comparison with measurements (points). In the dashed curve, the heating by the helicon wave has been neglected.

wave was evaluated using collisional theory [16] and the measurements of wave amplitude shown below [9]. The loss terms $Q_{\text {elas }}$ and $Q_{\text {inel }}$ arise from elastic and inelastic (excitation and ionization) collisions, respectively, with neutrals and ions, and $Q_{\perp}$ includes all losses in the radial direction. $Q_{\perp}$ is negligibly small, and the sum of $Q_{\text {elas }}$ and $Q_{\text {inel }}$ is given by the function $\varepsilon_{c}\left(T_{e}\right)$, evaluated by Vahedi [17], multiplied by the ionization frequency $N n\langle\sigma v\rangle_{\text {ioniz }}$, where $N$ is the neutral density, and $n(z)$ is the measured plasma density. Thus the right-hand side of equation (1) is a known function of $T_{e}$, and equations (1) and (2) take the form

$$
\partial^{2}\left(T_{e}^{7 / 2}\right) / \partial z^{2}=f\left(T_{e}\right) .
$$

Equation (3) was numerically integrated starting with the measured value of $T_{e}$ at the peak $(z=16 \mathrm{~cm})$, subject to the boundary condition that the slope $\partial T_{e} / \partial z$ be zero at large distances (actually, at the last data point). This procedure accurately determines the initial slope and the subsequent decay of $T_{e}$. The result is compared with the experimental data in figure 5, showing an extremely good fit. The two curves in figure 5 differ in the inclusion of $Q_{\text {wave }}$; it is seen that downstream heating by the wave has very little effect on the temperature profile.

From the initial slope, one can calculate with equation (2) the heat flow crossing the plane $z=16 \mathrm{~cm}$. This calculation is insensitive to the radial profiles, since $q$ is independent of $n$, and $T_{e}$ was observed to be nearly uniform across the diameter. The result is a heat flux of $\sim 1.4 \mathrm{~kW}$. Thus, approximately $70 \%$ of the $2 \mathrm{~kW}$ of $\mathrm{RF}$ power deposited into the plasma flows downstream and is dissipated by excitation of line radiation.

\subsection{Density profile}

In steady state, the net force on the electron fluid along $\boldsymbol{B}_{0}$ must be zero, since there are no magnetic forces in that 


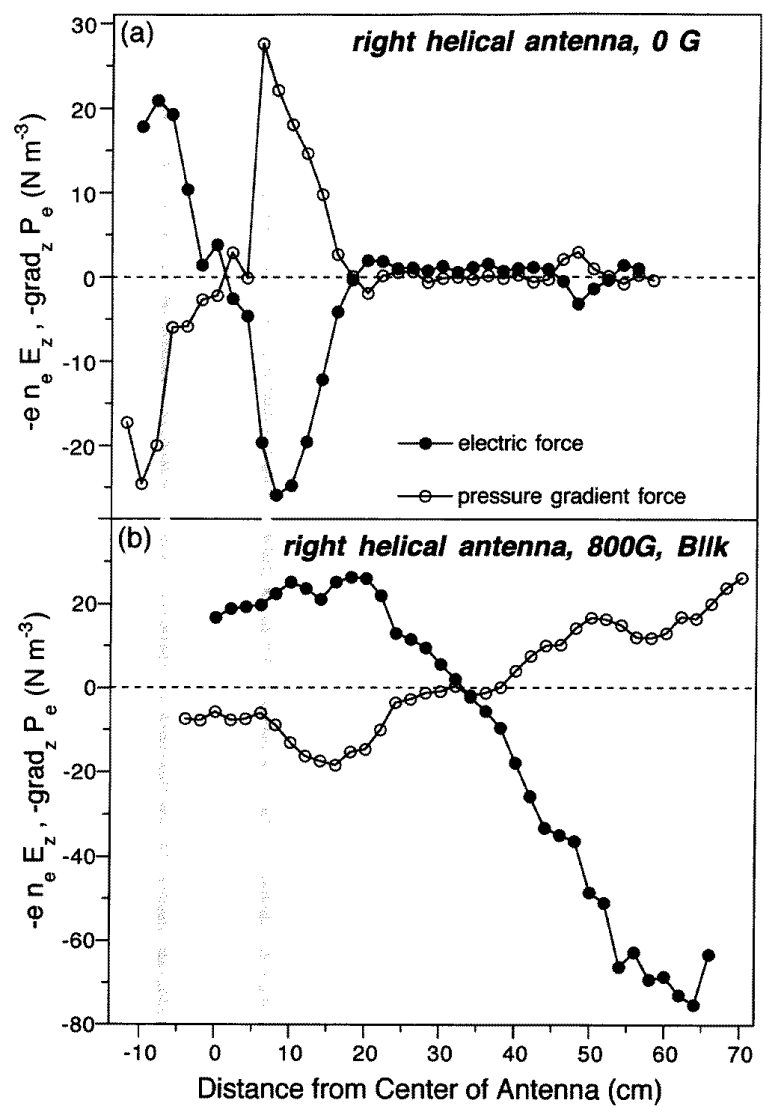

Figure 6. The electric and pressure gradient forces on the electron fluid for (a) a 0 G ICP discharge and (b) an 800 G helicon discharge, showing approximate pressure balance.

direction to restrain the electrons. The force equation in the $z$ direction is

$$
-e n E_{z}-\partial\left(n K T_{e}\right) / \partial z-m n v_{e} u_{z}+F_{N L}=0 .
$$

The collision term is negligibly small, since quasineutrality requires the electron fluid velocity $u_{z}$ to be no larger than an ion velocity. The ponderomotive force $F_{N L}$ along $\boldsymbol{B}_{0}$ is also negligible, since the wave magnetic field is only of the order of a few gauss. Thus, if we assume for the moment that $E_{z}$ is small because of high conductivity, the pressure gradient must also be small. As $T_{e}$ decays downstream, the density must rise to keep $n K T_{e}$ approximately constant. Ultimately, of course, the density must decay at large $z$ because ions cannot be transported from upstream, where they are created, as fast as they are lost to the walls. In practice, the $E_{z}$ term cannot be neglected and will be included below.

Pressure balance can be checked quantitatively by comparing the first two terms of equation (6), as is done in figure 6. For the ICP discharge at $0 \mathrm{G}$ (figure 6(a)) it is seen that the electric and pressure gradient forces, computed from the measured profiles of $V_{s}, n$ and $T_{e}$, are equal and opposite, as expected. For the $800 \mathrm{G}$ helicon discharge (figure 6(b)) the forces are opposite everywhere, but they are not quite equal in magnitude, especially in the downstream region. We believe, however, that pressure

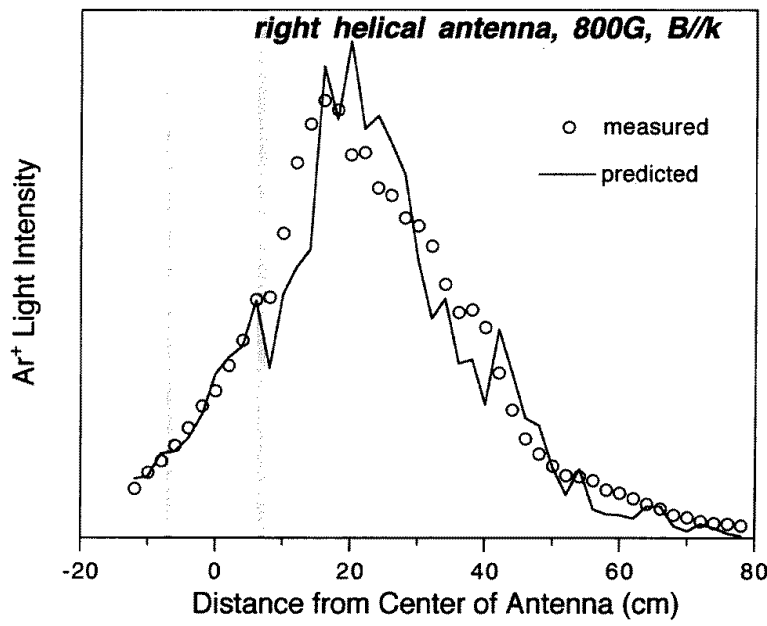

Figure 7. Comparison of the axial profile of light emission as observed (points) and calculated (line) from the measured density and electron temperature.

balance is at least approximately obeyed everywhere, and that the discrepancy arises from errors in RF compensation in the low-density region and in the interpretation of space potential measurements in a strong magnetic field. The density rise in the downstream region is caused by the decay in electron temperature and the need to compensate for the consequent decrease in electron pressure. During the set-up period for this equilibrium, the ions must move to their required positions. It would be impossible to predict the density distribution starting from ionization and ion diffusion considerations, since the electric fields which cause the ions to move downstream during the approach to equilibrium arise from small differences in the motion of the ion and electron fluids.

\subsection{Light emission}

Having measured the axial profiles of $T_{e}$ and $n$, we can calculate the expected axial profile of the $488 \mathrm{~nm} \mathrm{Ar}^{+}$line, assuming that the excitation is primarily from the ground level of the ion. As shown in figure 7, the result is in good agreement with the optical emission data. Further details are given in [11].

\section{Particle and energy balance}

Although the wave apparently generates little heat downstream, plasma is still being ionized there by the tail of the thermal electron distribution, and one must be sure that the production of ions there is consistent with the losses. The production rate can be calculated from the local temperature and density and the known ionization cross section for Ar. The plasma is assumed to be lost by radial ambipolar diffusion to the wall. The losses parallel to $B_{0}$ can be neglected because of the large aspect ratio of the tube. The local ambipolar diffusion coefficient $D_{a}$ can be calculated at each $z$ from the measured $T_{e}$ profile. The controlling process is the scattering of the magnetized electrons by the ions near the axis, and by the neutrals near 
(a)
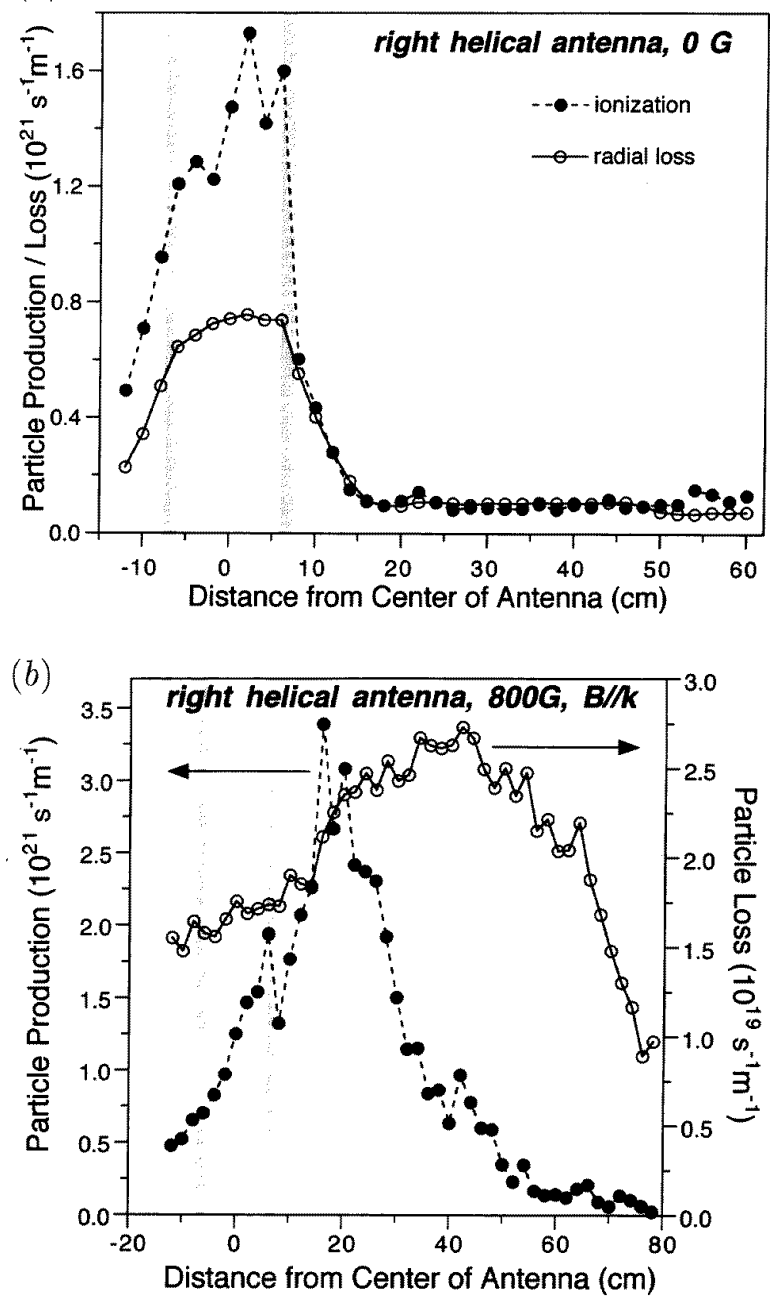

Figure 8. Calculated axial distributions of particle production (solid circles) and radial loss (open circles) for (a) a 0 G ICP discharge and (b) an 800 G helicon discharge.

the edge, where the ion density is low. The radial flux $-D_{a} \nabla n$ at the edge is then computed from the measured $n(z)$ assuming that the profile $n(r)$, measured at one value of $z$, does not change with $z$. Figure 8(a) shows the ion production and loss rates as functions of $z$ for the $0 \mathrm{G}$ ICP discharge. Both are peaked in the antenna region, and there is almost no plasma either upstream or downstream. The integrated production and loss rates differ by about a factor of 3; this is reasonable agreement in view of the uncertainties in this type of calculation. Figure 8(b) shows the production and loss rates for the $800 \mathrm{G}$ helicon discharge. Here the ionization occurs upstream, peaking where $T_{e}$ is high, but the losses occur mainly downstream, where the density is high and the electrons are more collisional. Magnetic confinement permits the plasma to be lost downstream from where it is produced. However, the integrated ionization rate is almost two orders of magnitude higher than the integrated loss rate, even though ionization by a fast electron population, if any, has been neglected.

This discrepancy is removed by considering the

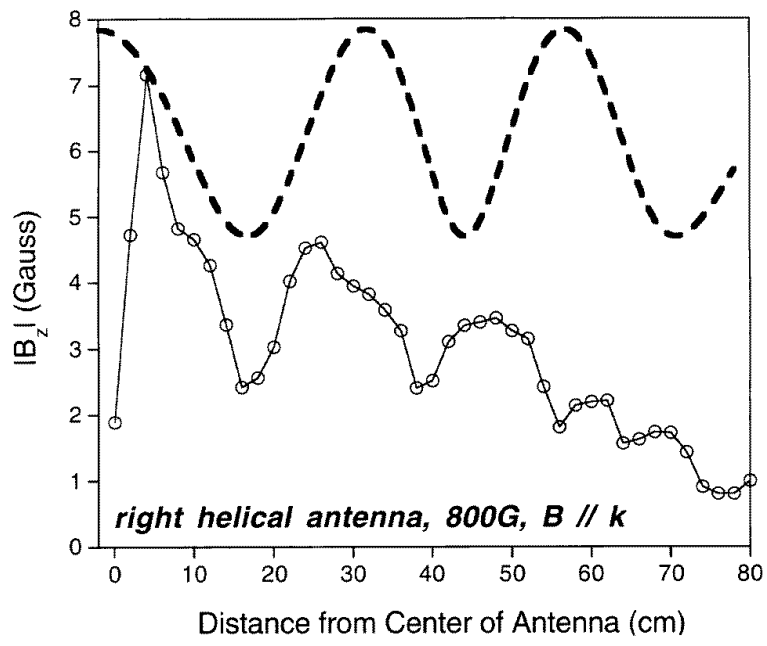

Figure 9. Magnitude of the $B_{z}$ component of the helicon wave field measured with a magnetic probe at $r / a=0.4$. The dashed line shows the computed beat wavelength of the two helicon modes expected to be dominant in the discharge.

decrease in neutral pressure that necessarily occurs in the high-density helicon mode because of the ion pumping effect. The discharge is pulsed, but the pulse length of $\sim 150 \mathrm{~ms}$ is long enough for the neutral gas to reach its equilibrium pressure. Neutral gas normally flows to the pump end of the discharge at room temperature; but when the $\mathrm{Ar}$ atoms are ionized, the presheath potential accelerates the ions, and they leave at the ion acoustic velocity $\left(K T_{e} / m\right)^{1 / 2}$, while the gas feed is unchanged. Without considering gradients in the neutral pressure, we estimate that the equilibrium pressure during the discharge is lowered by a factor of about 4 . Taking this into account, we gain a factor of 4 each in the ionization rate and the edge diffusion rate, bringing the production and loss rates to within a factor of 3 , as in the $0 \mathrm{G}$ case.

Having calculated the integrated ion loss rate, we can also estimate the radiation losses, since the number of electron-volts expended in creating each electron-ion pair is the known function $\varepsilon_{c}\left(T_{e}\right)$ used earlier [17]. When the kinetic energy carried out by escaping ions and electrons is added to this, we find that the integrated energy loss rate is within a factor of 2 of the total input power of $2 \mathrm{~kW}$ for both the ICP and helicon discharges.

\section{Behaviour of the helicon waves}

The axial variation of the $B_{z}$ component of the wave field was measured [10] with a balanced, 5-turn magnetic probe with its long, shielded leads terminating in a balun transformer.

\subsection{Modulation of the wave amplitude}

Figure 9 shows the axial variation of the magnitude of $B_{z}$ with the R-antenna; a 'standing wave' pattern is clearly seen. The pattern is reproducible to within the diameter of the data points but cannot be caused by standing 


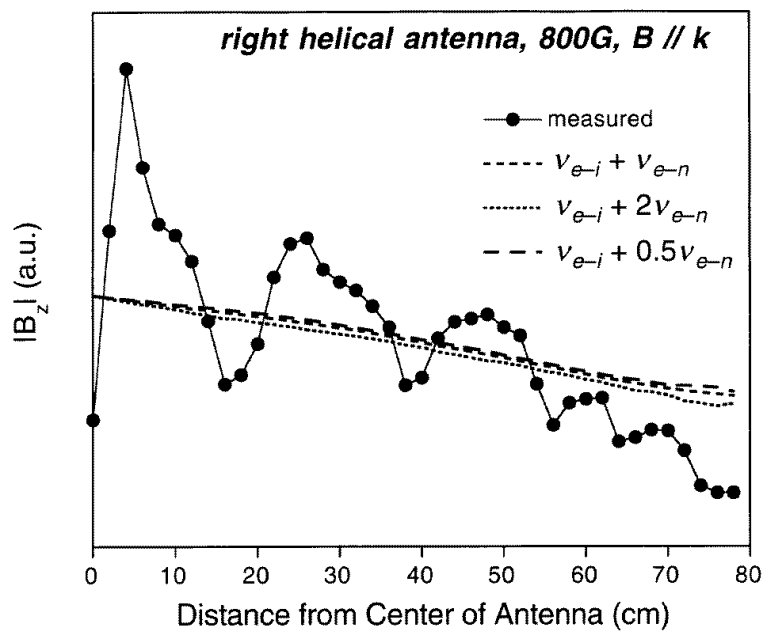

Figure 10. The data of figure 9 compared with the calculated decay in wave amplitude from collisional damping. The dashed lines show the effect of varying the neutral pressure a factor of 2 in either direction.

waves because of the small wave amplitude at the end where the waves could be reflected. The beat pattern can be explained, however, if we assume that different helicon modes are excited simultaneously by the antenna. Examination of the $k$ values corresponding to the axial wavelengths of various helicon modes revealed that only the first and second radial modes $(n=1,2)$ of the $m=+1$ azimuthal mode had $k$ values falling within the largest peak of the $k$-spectrum of the antenna current distribution. Assuming that the $(m, n)=(1,1)$ and $(1,2)$ modes are excited with an amplitude ratio calculated from the depth of the observed modulations, we computed the $k$ values of these two modes taking into account the measured radial and axial plasma density profiles. The resulting beat pattern is shown by the dashed line in figure 9 . The spacing between minima is consistent with the two-mode hypothesis, though evidence for other modes can be seen far from the antenna. In this calculation the damping of each mode was not taken into account because the damping rate varies with radius, and this effect has not yet been treated theoretically.

\subsection{Wave damping rate}

The general damping rate for $\left|B_{z}\right|$, averaged over the amplitude modulations, was calculated from uniformplasma theory [16] with a radially averaged density. Figure 10 shows that electron-ion collisions alone accounts for essentially all of the damping we observed.

\subsection{Wavelength variation}

The phase of $B_{z}$ was also measured, using a fixed probe as a reference. The local wavelength was found to change with $z$, as shown in figure 11 . That the wavelength should vary inversely with density is expected from the basic dispersion relation for helicon waves,

$$
\alpha=\frac{\omega}{k} \frac{n_{0} e \mu_{0}}{B_{0}} \propto \frac{1}{a}
$$

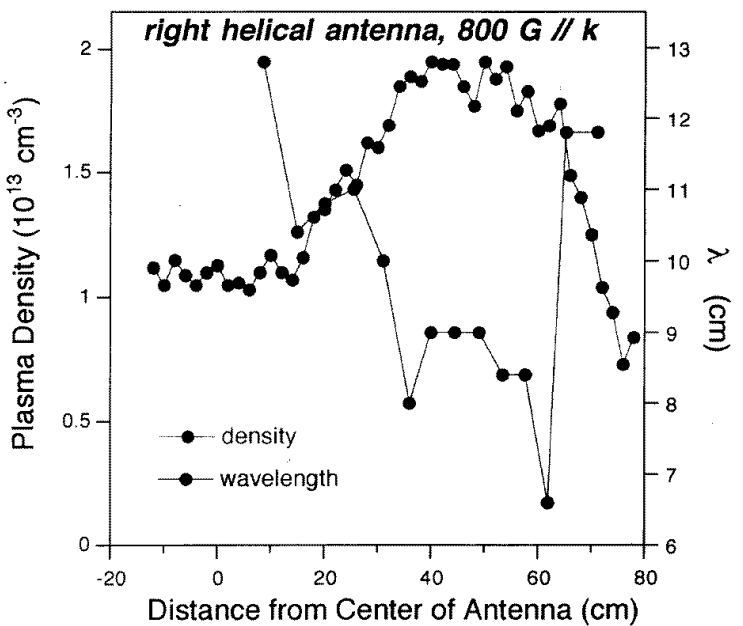

Figure 11. Measurements of the local wavelength of the helicon wave, showing its inverse relation to the local density (top curve).

where $\alpha$ is the total wavenumber and $a$ the plasma radius. However, the magnitude of the wavelength itself is about half that expected for a half-wavelength antenna. The reason for this, we believe, is that the end-rings of the helical antenna, which are necessary for the current return, generate a $k$-spectrum with a large component at the shorter wavelength.

\section{Conclusions}

Detailed measurements of the downstream plasma and wave characteristics of a helicon discharge show that

(a) Helicon wave propagation is needed for the large density increase over the ICP,

(b) The $m=+1$ mode is preferentially excited,

(c) The RF energy is almost all absorbed in the nearfield of the antenna rather than downstream,

(d) The temperature decays downstream as predicted by heat conduction and inelastic electron collisions,

(e) An unexpected density peak far from the antenna can be explain by pressure balance,

(f) Particle and power balance in the downstream region is roughly in agreement with theory,

(g) The effect of neutral depletion by ion pumping is strong in high-density discharges and

(h) The wave field behaviour downstream follows theory, except that an unexpected beating pattern is observed and is consistent with the simultaneous launching of the two dominant helicon modes.

For use of helicon discharges in semiconductor processing, these results imply that

(a) The RF energy can be absorbed efficiently in a short discharge and

(b) A long discharge can produce the low-temperature, high-density conditions suitable for negative-ion etching in 'afterglow' conditions or for producing the un-ionized, active species for chemical downstream etching. 
Our attempts to observe kinetic effects have failed to reveal the existence of a high-energy electron tail caused by Landau damping. Indeed, all of our results can be explained by collisional theory alone. However, there is still a possibility that the efficient near-field absorption of RF energy is related to wave acceleration of electrons; this region was outside the scope of this work.

\section{Acknowledgments}

This work was supported by the Semiconductor Research Corporation, Contract No 95-IJ-529, the National Science Foundation, Grant No ECS 94-00849, the Wisconsin Engineering Research Center for Plasma-Aided Manufacturing, and the Lawrence Livermore Laboratory Plasma Physics Research Institute. We acknowledge the help of D D Blackwell and D Arnush throughout this work.

\section{References}

[1] Nakano T, Gottscho R A, Sadeghi N, Trevor D J, Boswell R W, Perry A J, Lee T C, Giapis K P and Margot J 1992 Helicon wave excited plasmas Oyo Buturi (Japan. Soc. Appl. Phys.) 61711
[2] Lieberman M A and Gottscho R A 1994 Design of high density plasma sources for materials processing Physics of Thin Films (New York: Academic)

[3] Chen F F 1996 Helicon plasma sources High Density Plasma Sources ed Oleg A Popov (Park Ridge, NJ: Noyes Publications) to be published

[4] Chen F F and Chevalier G 1992 J. Vac. Sci. Technol. A 10 1389

[5] Bailey A D III 1995 private communication

[6] Samukawa S, Kinoshita K and Mieno T 1996 Plasma Sources Sci. Technol. 5

[7] Ahn T H, Nakamura K and Sugai H 1996 Plasma Sources Sci. Technol. 5

[8] Chevalier G and Chen F F 1993 J. Vac. Sci. Technol. A 11 1165

[9] Light M and Chen F F 1995 Phys. Plasmas 21084

[10] Light M, Sudit I D, Chen F F and Arnush D 1995 Phys. Plasmas 24094

[11] Sudit I D and Chen F F 1995 Discharge equilibrium of a helicon plasma Plasma Sources Sci. Technol. 543

[12] Chen F F, Hsieh M J and Light M 1994 Plasma Sources Sci. Technol. 349

[13] Sudit I D and Chen F F 1994 Plasma Sources Sci. Technol. 3602

[14] Sudit I D and Chen F F 1994 Plasma Sources Sci. Technol. 3162

[15] Ellingboe A 1996 Plasma Sources Sci. Technol. 5

[16] Chen F F 1995 Plasma Phys. Controlled Fusion 33339

[17] Vahedi V 1994 Principles of Plasma Discharges and Materials Processing ed M A Lieberman and A J Lichtenberg (New York: Wiley) p 81 